\title{
Selective Eradication of Staphylococcus aureus by the Designer Genetically Programmed Yeast Biocontrol Agent
}

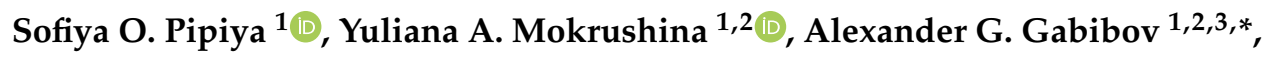 \\ Ivan V. Smirnov ${ }^{1,2, *}$ and Stanislav S. Terekhov ${ }^{1,2, *(D)}$ \\ 1 Shemyakin-Ovchinnikov Institute of Bioorganic Chemistry, Russian Academy of Sciences, Moscow 117997, \\ Russia; sonya-pipiya@yandex.ru (S.O.P.); yuliana256@mail.ru (Y.A.M.) \\ 2 Department of Chemistry, Lomonosov Moscow State University, Moscow 119991, Russia \\ 3 Department of Life Sciences, Higher School of Economics, Moscow 101000, Russia \\ * Correspondence: gabibov@ibch.ru (A.G.G.); smirnov@ibch.ru (I.V.S.); sterekhoff@gmail.com (S.S.T.)
}

Received: 17 July 2020; Accepted: 18 August 2020; Published: 19 August 2020

\begin{abstract}
Staphylococcus aureus is a common human pathogen that is particularly often associated with antibiotic resistance. The eradication of this ubiquitous infectious agent from its ecological niches and contaminated surfaces is especially complicated by excessive biofilm formation and persisting cells, which evade the antibacterial activity of conventional antibiotics. Here, we present an alternative view of the problem of specific $S$. aureus eradication. The constitutive heterologous production of highly specific bacteriolytic protease lysostaphin in yeast Pichia pastoris provides an efficient biocontrol agent, specifically killing $S$. aureus in coculture. A yeast-based anti-S. aureus probiotic was efficient in a high range of temperatures and target-to-effector ratios, indicating its robustness and versatility in eliminating $S$. aureus cells. The efficient eradication of $S$. aureus by live lysostaphin-producing $P$. pastoris was achieved at high scales, providing a simple, biocompatible and cost-effective strategy for S. aureus lysis in bioproduction and surface decontamination. Future biomedical applications based on designer yeast biocontrol agents require evaluation in in vivo models. However, we believe that this strategy is very promising since it provides highly safe, efficient and selective genetically programmed probiotics and targeted biocontrol agents.
\end{abstract}

Keywords: Staphylococcus aureus eradication; yeast biocontrol agent; recombinant lysostaphin; genetically programmed probiotics; targeted lysis; constitutive heterologous production in Pichia pastoris

\section{Introduction}

Antibiotic resistance (AR) is a persistent threat to global healthcare, resulting in up to 11 million deaths annually $[1,2]$. Staphylococcus aureus is one of the most common pathogens, accounting for nearly 120,000 severe cases of bloodstream infections and 20,000 deaths per year in the United States alone [3]. S. aureus is a highly specialized human pathogen, notorious for an extensive number of methicillin-resistant (MRSA) strains resistant to the entire $\beta$-lactam antibiotic class [4]. Moreover, S. aureus strains have adopted a variety of virulence factors to efficiently colonize and counteract host immunity [5]. The exceptionally flexible $S$. aureus adaptation to antibiotics arises not only from the horizontal transfer of mobile genetic elements. It is also enhanced by increased mutation rates of $S$. aureus strains. The estimated mutation rate for the $S$. aureus isolate collection is $3.3 \times 10^{-6}$ per site per year [6]. This rate is about 1000 times faster than the canonical substitution rate estimate for E. coli [7]. A high mutation rate of S. aureus isolates relates to their low doubling time in the 
wild [8]. This mutability provides the increased evolutionary space for S. aureus that it implements for its rapid adaptation. Hence, the eradication of $S$. aureus is an incredibly difficult task because of its plasticity, ubiquity, propensity to AR and simplicity of transmission [5]. Extensive biofilm formation and persisters in turn worsen the situation dramatically, making $S$. aureus non-sensitive to antibiotic drugs [9].

Bacteriolytic enzymes, disrupting cell integrity, represent a promising alternative to conventional antibiotics. The most promising example of the former is lysostaphin, which not only kills planktonic S. aureus but also disrupts S. aureus biofilms [10]. Lysostaphin is a specific zinc-containing metalloprotease secreted by strains of $S$. simulans biovar staphylolyticus [11]. Lysostaphin cleaves the pentaglycine crossbridges present in peptidoglycan (PG), the essential component of the bacterial cell wall, leading to rapid cell lysis. The recognition of the pentaglycine crossbridge and the peptide stem is shared by two independent SH3b domains, allowing protein clustering on the PG [12]. This unusual mechanism underpins the potent activity of lysostaphin, and its capacity to punch holes in the cell walls so as to cause rapid cell lysis.

Lysostaphin displays bacteriolytic activity against numerous Staphylococcus species [13,14]. It is highly active against virtually any natural $S$. aureus strain, including clinically relevant MRSA and vancomycin intermediately susceptible S. aureus (VISA) $[15,16]$. This makes lysostaphin a promising agent in the experimental therapy of $S$. aureus infections overperforming conventional vancomycin therapy in different animal models [17-19], thus providing an essential therapeutic agent against VISA and vancomycin-resistant $S$. aureus (VRSA) [20].

A wide palette of applications illustrates the versatility and efficiency of lysostaphin in the eradication of $S$. aureus from different environments. Hydrogel-based materials eliminating implant S. aureus infections represent an example of an advanced biomaterial facilitating healing [21]. The heterologous production of lysostaphin was applied to protect transgenic animals from mastitis, eradicating S. aureus from milk and mammary tissue [22]. Encapsulated mammalian cells with a synthetic gene circuit to sense and respond to MRSA infection could provide potential prophylactic, diagnostic or therapeutic options for treating medical implant-associated infections [23]. Deimmunized lysostaphin variants are valuable in this case as they expand the boundaries of lysostaphin systemic administration and therapy [24].

Here we describe an alternative paradigm of specific $S$. aureus eradication. We consider applying the high viability, robustness, and safety of yeasts to create a genetically programmed biocontrol agent, killing S. aureus cells and destroying its biofilms. The heterologous inducible production of recombinant lysostaphin in yeast Pichia pastoris was previously achieved via the codon optimization of its natural sequence [25]. Alternatively, we focused on the constitutive production of lysostaphin in yeast cells to obtain live cell cultures, eradicating S. aureus. P. pastoris produced up to $30 \mathrm{mg} / \mathrm{L} /$ day of recombinant lysostaphin in shaking flask conditions. This culture medium was highly active against $S$. aureus, leading to growth inhibition and bactericidal effects at $1.6 \times 10^{4}$ - and $4 \times 10^{3}$-fold dilutions, respectively. Moreover, $10^{7} \mathrm{CFU} / \mathrm{mL}$ of $P$. pastoris eradicated up to $10^{10} \mathrm{CFU} / \mathrm{mL}$ S. aureus after $12 \mathrm{~h}$ in coculture. Recombinant lysostaphin-producing (rLys) P. pastoris was active against S. vitulinus and S. xylosus, which have similar PG crossbridge sequences, correlating with the lysostaphin activity spectrum. The anti-S. aureus activity of live rLys P. pastoris culture was prominent at the $20-33^{\circ} \mathrm{C}$ temperature range, with some decrease at $37^{\circ} \mathrm{C}$ that we associate with the elevated growth temperature, non-optimal for P. pastoris. The efficient eradication of $S$. aureus via engineered $P$. pastoris in coculture was achieved in a broad range of target (S. aureus):effector (rLys P. pastoris) ratios, both at high and low S. aureus cell densities. These results illustrate the considerable potential of live lysostaphin-producing cultures in S. aureus elimination, creating a background for their further applications in vivo. Yeast-based bacteriolytic live cultures represent a particularly promising approach to the creation of highly safe, efficient and selective genetically programmed probiotics and targeted designer biocontrol agents. 


\section{Results}

\subsection{Constitutive Production of Recombinant Lysostaphin in P. pastoris}

The codon-optimized sequence of lysostaphin [25] was cloned into pGAP $\alpha$ under control of the strong constitutive glyceraldehyde-3-phosphate dehydrogenase (GAP) promoter, so as to maintain constitutive production of the recombinant lysostaphin in P. pastoris (Figure 1A). The P. pastoris transformed with $\mathrm{pGAP} \alpha$-Lys vector produced recombinant lysostaphin, and secreted it into the culture medium, giving clear zones of $S$. aureus inhibition in an agar overlay assay (Figure 1B).

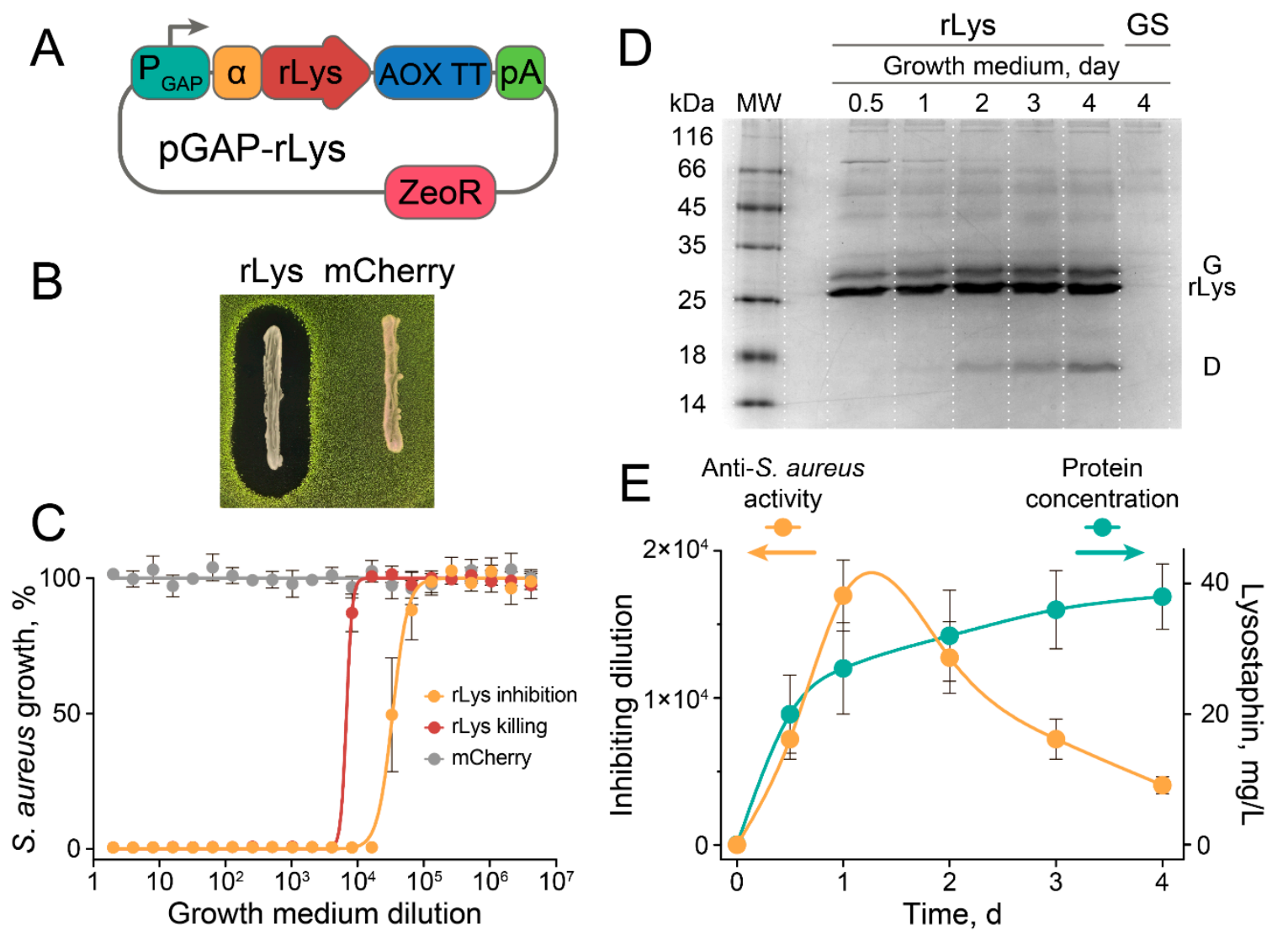

Figure 1. Production of recombinant lysostaphin (rLys) in P. pastoris and its anti-S. aureus activity. (A) Schematic representation of vector $\mathrm{pGAP} \alpha$-Lys engineered for heterologous constitutive production of rLys in P. pastoris. $\mathrm{P}_{\mathrm{GAP}}$-glyceraldehyde-3-phosphate dehydrogenase (GAP) promoter, $\alpha$-alpha-factor signal sequence, rLys—codon-optimized sequence of lysostaphin, AOX TT_AOX1 transcriptional terminator, $\mathrm{pA}$ - polyadenylation signal, ZeoR—zeocin resistance. (B) Agar overlay assay of P. Pastoris GS115 transformed with pGAP $\alpha$-Lys (rLys) or control vector pGAP $\alpha$-mCherry (mCherry). GFP-producing S. aureus was used as an indicator strain. (C) Inhibition of S. aureus growth by rLys $P$. pastoris culture medium. S. aureus growth was estimated by the fluorescence of GFP reporter. rLys inhibition (orange) and rLys killing (red) was measured after 1 day and 1 week, respectively. mCherry (gray) indicates the control medium obtained from mCherry-producing P. Pastoris. (D) rLys daily production into the growth medium, visualized by 15\% PAGE. G—glycosylated rLys; D—a byproduct of rLys proteolytic degradation; GS- parent P. pastoris GS115 strain. rLys production was monitored in rLys P. Pastoris culture supernatant. (E) Anti-S. aureus activity in culture medium (orange) and protein production (aquamarine) time curves. Data represent the mean of three biological replicates $\pm \mathrm{SD}$.

The culture medium of rLys $P$. pastoris was highly active against $S$. aureus, leading to growth inhibition and bactericidal effects at $1.6 \times 10^{4}$ - and $4 \times 10^{3}$-fold dilutions, respectively (Figure 1C). 
Control P. pastoris strain producing mCherry fluorescent protein did not influence $S$. aureus growth and viability (Figure 1B,C). P. pastoris produced up to $30 \mathrm{mg} / \mathrm{L} /$ day of recombinant lysostaphin in shaking flask conditions (Figure 1D,E), and we estimate that this productivity could be increased by several folds using bioreactor cultivation conditions. However, proteolysis of the recombinant lysostaphin was observed (Figure 1D), resulting in the maximal anti-S. aureus activity detected at the $24 \mathrm{~h}$ cultivation time point (Figure 1E). Since the specific activity of the rLys P. pastoris culture medium gradually decreased after $24 \mathrm{~h}$ of cultivation, we speculate that the production of recombinant lysostaphin by live rLys P. pastoris culture in situ is preferred for enhanced S. aureus eradication.

\subsection{The Anti-Stapylococcal Activity of Engineered rLys P. pastoris}

Unlike a parent P. pastoris GS115 strain, engineered rLys P. pastoris displays antagonistic properties, mediated by constitutive lysostaphin production. To trace the correlation between lysostaphin activity spectrum and the antagonistic properties of rLys P. pastoris against different bacteria, we used a panel of Staphylococci and several other bacteria naturally resistant toward lysostaphin (Figure 2). The antagonistic properties of rLys P. Pastoris were similar to the lysostaphin activity spectrum. The most efficient growth inhibition was observed for S. aureus and S. vitulinus. Mediocre inhibition was observed for S. xylosus. Several Staphylococci (Staphylococcus pasteuri, Staphylococcus warneri, Staphylococcus haemolyticus and Staphylococcus epidermidis) and other bacteria (Macrococcus caseolyticus, Bacillus subtilis, Enterococcus faecium, Enterococcus faecalis, and Escherichia coli) were not inhibited by rLys $P$. pastoris. This is in line with the lysostaphin activity spectrum, and it is associated with the alternative PG structures among these bacteria [26].

\begin{tabular}{|c|c|c|c|c|c|}
\hline \multirow[b]{2}{*}{ Bacterium } & \multicolumn{2}{|c|}{ Medium } & \multirow{2}{*}{$\begin{array}{c}\text { Coculture } \\
\text { Inhibiting } \\
\text { rLys } P \text {. pastoris, } \\
\text { CFU } / \mathrm{mL}\end{array}$} & \multicolumn{2}{|c|}{ Microscopy } \\
\hline & $\begin{array}{l}\mathrm{MIC} \\
\mathrm{ng} / \mathrm{mL}\end{array}$ & $\begin{array}{l}\mathrm{MBC} \\
\mathrm{ng} / \mathrm{mL}\end{array}$ & & $\begin{array}{c}\text { rLys } \\
\text { P. pastoris }\end{array}$ & $\begin{array}{l}\text { mCherry } \\
\text { P. pastoris }\end{array}$ \\
\hline S. aureus & $2 \pm 1$ & $8 \pm 4$ & $2 \pm 1 \times 10^{5}$ & & \\
\hline S. vitulinus & $4 \pm 2$ & $20 \pm 8$ & $2 \pm 1 \times 10^{5}$ & & \\
\hline S. xylosus & $8 \pm 4$ & $300 \pm 130$ & $3 \pm 1.6 \times 10^{6}$ & 64 & \\
\hline
\end{tabular}

Figure 2. Antibacterial activity of rLys produced in a culture medium, and inhibitory activity of live rLys $P$. pastoris cell culture. The growth medium was collected after $1 \mathrm{~d}$ of cultivation. Live rLys $P$. pastoris cells were washed with a fresh medium before cocultivation with bacteria. $\sim 10^{6} \mathrm{CFU} / \mathrm{mL}$ of indicator bacteria was used. Staphylococcus pasteuri, Staphylococcus warneri, Staphylococcus haemolyticus, Staphylococcus epidermidis, Macrococcus caseolyticus, Bacillus subtilis, Enterococcus faecium, Enterococcus faecalis and Escherichia coli were not inhibited by rLys P. pastoris and rLys up to $50 \mu \mathrm{g} / \mathrm{mL}$ concentration. MIC - minimum inhibitory concentration, MBC—-minimum bactericidal concentration.

Hence, engineered rLys P. pastoris displays quite a narrow activity spectrum. rLys P. pastoris acts efficiently on S. aureus and several Staphylococci, these having similar PG structures. This selectivity is favored for the practical application of rLys P. pastoris as it could be exploited as a targeted biocontrol agent for $S$. aureus eradication.

\subsection{Eradication of S. aureus by Live rLys P. pastoris Biocontrol Agent}

Focusing on the inhibitory properties of rLys P. pastoris cells alone, we screened different cocultivation modes of S. aureus and rLys P. pastoris. S. aureus and P. pastoris have different optimal 
growth temperatures, $37^{\circ} \mathrm{C}$ and $30^{\circ} \mathrm{C}$, respectively. Hence, elevated temperatures could decrease the anti-S. aureus activity of rLys $P$. pastoris (Figure 3 ).

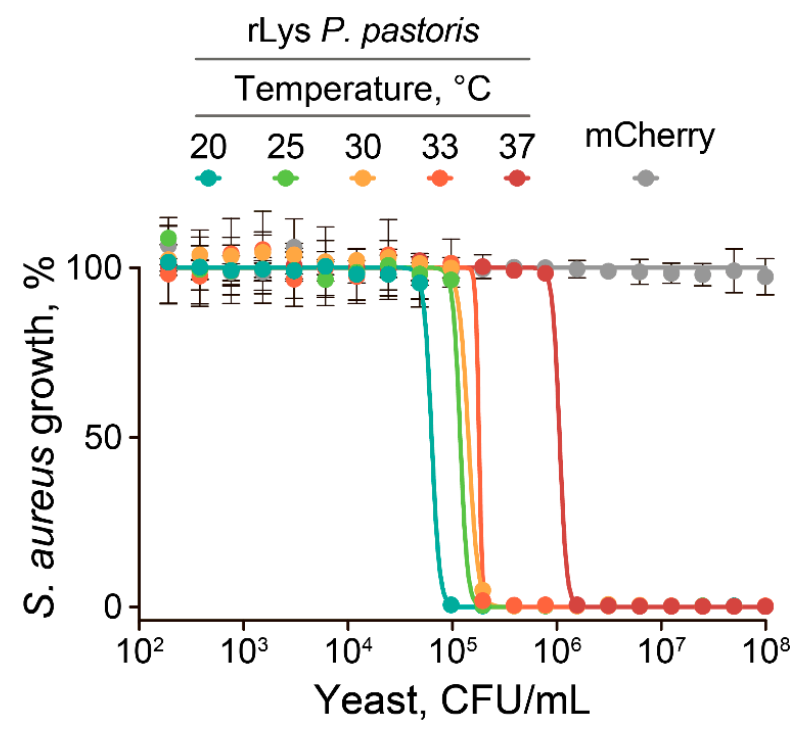

Figure 3. Growth temperature influences the inhibitory activity of the live rLys P. pastoris cell culture. The cocultivation of rLys $P$. pastoris with a GFP-producing $S$. aureus indicator strain was performed at different temperatures in the $20-37^{\circ} \mathrm{C}$ range. S. aureus growth was estimated by the fluorescence of the GFP reporter. mCherry (gray) indicates control mCherry-producing P. pastoris strain. Data represent the mean of three biological replicates \pm SD.

However, efficient S. aureus inhibition by rLys P. pastoris was observed up to $33^{\circ} \mathrm{C}$, corresponding to the temperature of the skin surface, which is an ecological niche of S. aureus. The further increase in cocultivation temperature resulted in an eight-fold decrease of anti-S. aureus activity at $37^{\circ} \mathrm{C}$ (Figure 3 ). S. aureus inhibition was particularly efficient at temperatures below $25^{\circ} \mathrm{C}$, which is close to natural $P$. pastoris growth conditions.

To estimate the kinetics of S. aureus eradication, we varied the target (S. aureus):effector (rLys $P$. pastoris) ratios and measured cell viability after cocultivation at $33{ }^{\circ} \mathrm{C}$ (Figure 4 ).

At relatively high rLys $P$. pastoris loads $\left(10^{7} \mathrm{CFU} / \mathrm{mL}\right.$ up to $\left.10^{10} \mathrm{CFU} / \mathrm{mL}\right), S$. aureus cells were killed within $12 \mathrm{~h}$ of cocultivation (Figure $4 \mathrm{~A}$ ). The control P. pastoris strain producing the mCherry fluorescent protein did not influence $S$. aureus viability, as was expected from the culture medium activity testing (Figure 4A). Decreasing the load of rLys P. pastoris to $10^{6} \mathrm{CFU} / \mathrm{mL}$ resulted in different scenarios. At a low S. aureus load, $10^{6} \mathrm{CFU} / \mathrm{mL}$, rLys P. pastoris controlled $S$. aureus growth and eliminated viable cells within less than $12 \mathrm{~h}$ (Figure $4 \mathrm{~B}$ ). Increasing the S. aureus loads to $10^{8} \mathrm{CFU} / \mathrm{mL}$ resulted in a situation wherein rLys $P$. pastoris could not control $S$. aureus growth from the beginning of cocultivation. This led to $S$. aureus proliferation at the $12 \mathrm{~h}$ time point, while after that rLys $P$. pastoris produced enough lysostaphin to kill the majority of $S$. aureus cells, decreasing its titer by 5.8 orders of magnitude after $24 \mathrm{~h}$ of cocultivation (Figure 4B). In dense $S$. aureus cultures with loads up to $10^{10} \mathrm{CFU} / \mathrm{mL}$, rLys $P$. pastoris gradually decreased $S$. aureus titer by 0.5 and 5.1 orders of magnitude at the $12 \mathrm{~h}$ and $24 \mathrm{~h}$ time points, respectively (Figure $4 \mathrm{~B}$ ). The decrease in viable S. aureus cells was associated with the massive $S$. aureus lysis detected by culture clearance (Figure 4C,D).

Thus, the complete or near-complete lysis of $S$. aureus cells is achieved by considerably low rLys $P$. pastoris concentrations at $12 \mathrm{~h}$. P. pastoris cultures reach cell densities exceeding $10^{10} \mathrm{CFU} / \mathrm{mL}$ when cultivated in bioreactors. Hence, more than 1000-fold dilutions of rLys P. pastoris live cell cultures are efficient in $S$. aureus eradication. This makes it a safe and cost-effective biocontrol agent for eliminating S. aureus cells.

To further estimate the eradication landscapes of the rLys P. pastoris biocontrol agent, we performed time-lapse cocultivation of S. aureus and rLys P. pastoris at different target:effector ratios (Figure 5). 
A

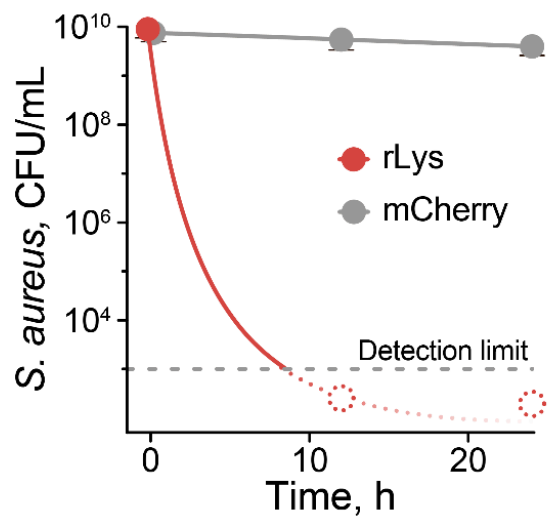

B

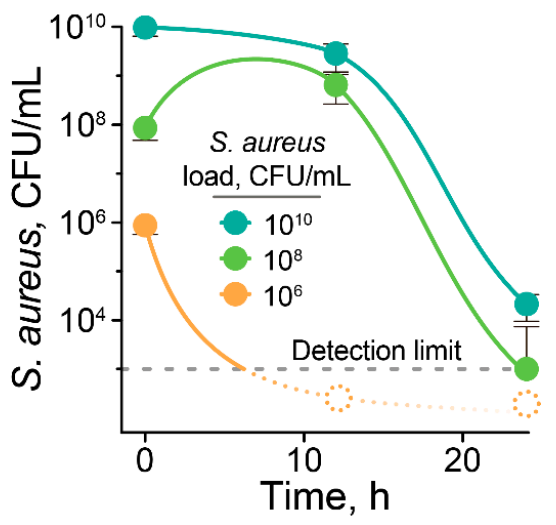

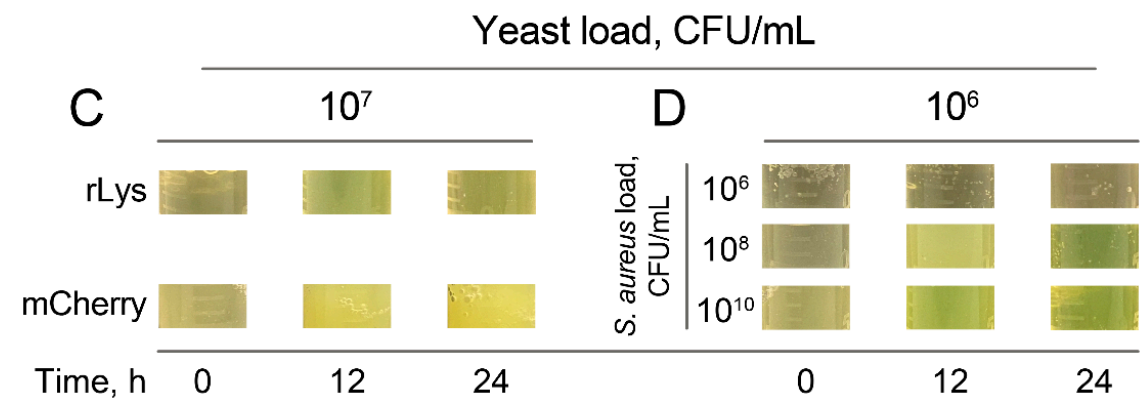

Figure 4. The cocultivation of S. aureus with rLys P. pastoris results in S. aureus killing. (A) Viability of S. aureus in coculture with $10^{7} \mathrm{CFU} / \mathrm{mL}$ of rLys P. pastoris (red) or control mCherry-producing P. pastoris (gray). Dashed dots indicate that no viable $S$. aureus was detected at the respective time point. The detection limit of viable $S$. aureus cells was $\sim 10^{3} \mathrm{CFU} / \mathrm{mL}$. (B) Viability of $S$. aureus in coculture with $10^{6} \mathrm{CFU} / \mathrm{mL}$ of rLys $P$. pastoris at various $S$. aureus loads. $10^{10}$ (aquamarine), $10^{8}$ (green) and $10^{6}$ (orange) CFU/mL of S. aureus were used as a starting point. Data represent the mean of three biological replicates \pm SD. $(\mathbf{C}, \mathbf{D})$ illustrate representative images of $S$. aureus/P. pastoris cocultures at various time points, corresponding to panels $(\mathbf{A}, \mathbf{B})$, respectively.

$25^{\circ} \mathrm{C}$ rLys $P$. pastoris, CFU/mL

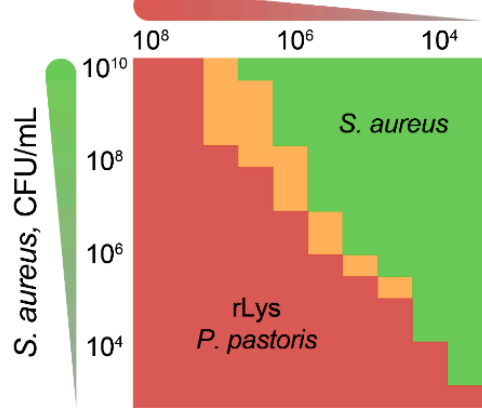

$33^{\circ} \mathrm{C}$

rLys $P$. pastoris, CFU/mL

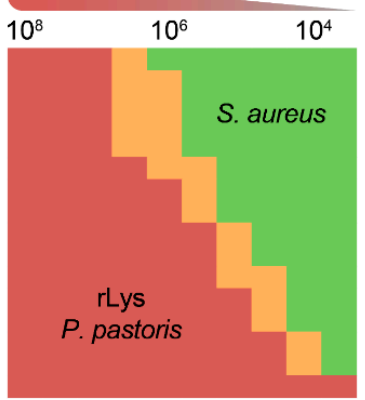

$37^{\circ} \mathrm{C}$

rLys $P$. pastoris, CFU/mL

$10^{8} \quad 10^{6} \quad 10^{4}$

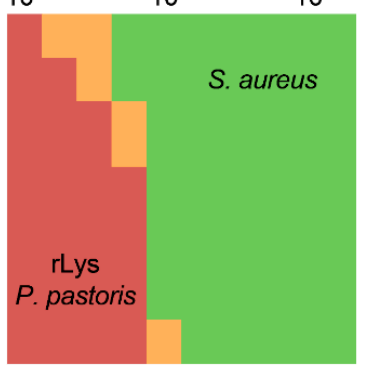

S. aureus

Survived

Eradicated after 5 days

Eradicated after 1 day

Figure 5. S. aureus eradication landscapes obtained by cocultivation with rLys P. pastoris. Various concentrations of $S$. aureus were cocultivated with rLys P. pastoris, resulting in S. aureus growth or eradication. Viable S. aureus cells were assayed after 1 day and 5 days. Areas indicating S. aureus/rLys $P$. pastoris ratios where live $S$. aureus was not detected after 1 day and 5 days are colored with red and orange, respectively. Areas with live S. aureus are colored with green. The detection limit of viable S. aureus cells is $\sim 10^{3} \mathrm{CFU} / \mathrm{mL}$.

rLys $P$. pastoris eradicated $S$. aureus at various target:effector ratios at temperatures from $25^{\circ} \mathrm{C}$ to $37^{\circ} \mathrm{C}$ (Figure 5). However, the eradication of $S$. aureus was not observed at low $\left(10^{6} \mathrm{CFU} / \mathrm{mL}\right)$ loads of rLys $P$. pastoris at $37^{\circ} \mathrm{C}$, indicating the necessity of effector proliferation for complete $S$. aureus 
removal (Figure 5). Both $25^{\circ} \mathrm{C}$ and $33^{\circ} \mathrm{C}$ temperatures were convenient for rLys P. pastoris growth resulting in $S$. aureus elimination even at yeast titers less than $10^{4} \mathrm{CFU} / \mathrm{mL}$. However, only up to $10^{4} \mathrm{CFU} / \mathrm{mL}$ of $S$. aureus could be eradicated at such a low yeast level. The temperature of $25^{\circ} \mathrm{C}$ resulted in a more efficient $S$. aureus eradication than $33^{\circ} \mathrm{C}$ at low yeast loads, while this relation was the opposite at high effector concentrations (Figure 5). We associate this with the counteraction of the more efficient proliferation of rLys P. pastoris at low temperatures, and the increased bacteriolytic activity at high temperatures.

\section{Discussion}

S. aureus is a ubiquitous human bacterium with outstanding pathogenic potential. Infections that are caused by AR S. aureus, particularly MRSA strains, result in epidemic waves still presenting a global threat $[4,5]$. Conventional antibiotics are often inefficient in S. aureus infections nowadays. Potent probiotics antagonizing $S$. aureus could be an alternative, or could provide synergistic effects in S. aureus eradication. Efficient S. aureus-killing bacteria are presented in bacterial communities of human [27] and animal [28,29] origins. However, the native properties of these killing bacteria may considerably hinder their application as a probiotic and a safe biocontrol agent in vivo, or even make it dangerous [27]. Designer genetically programmed probiotics, on the other hand, are free of these side effects. Here we suggest engineering anti-S. aureus yeasts, since they are safe, simple to manipulate, and generally easily tolerate stress conditions. Similar to the majority of non-sporulating bacteria, S. aureus displays a rapid decrease in cell viability under starvation, resulting in 99-99.9\% cell death after 2 days [30]. Unlike bacteria, yeasts have additional molecular pathways to adapt themselves to the various environmental conditions, e.g., introns promote yeast growth under starvation [31]. Hence, yeasts have an improved plasticity to survive under stress conditions and starvation, maintaining more than $50 \%$ survival for weeks and even months [32,33]. This is especially relevant to P. pastoris, which efficiently maintains viability by transcriptional reprogramming toward near-zero growth rates [34]. Thus, the genetically programmed S. aureus-killing yeast represents a proof-of-concept of the targeted designer yeast biocontrol agent.

rLys yeast efficiently eradicated S. aureus under a broad range of conditions and target:effector ratios. Even the 1000-fold numerical superiority of S. aureus over rLys P. pastoris in a dense culture resulted in S. aureus eradication. Moreover, complete $S$. aureus lysis could be achieved by cocultivation with only $10^{7} \mathrm{CFU} / \mathrm{mL}$ in less than $12 \mathrm{~h}$. Trace amounts of rLys P. pastoris eliminated similar concentrations of S. aureus in coculture at $25-33^{\circ} \mathrm{C}$. The optimal growth temperature for P. pastoris is $28-30{ }^{\circ} \mathrm{C}$. However, efficient $S$. aureus eradication was observed up to $33^{\circ} \mathrm{C}$. Even at $37^{\circ} \mathrm{C}$, the antagonistic effect of rLys $P$. pastoris was substantial. Although the reduction of $S$. aureus' virulence factors at colder temperatures may take place [35] and influence eradication efficacy, we attribute the decreased efficiency of rLys P. pastoris at $37^{\circ} \mathrm{C}$ to the impaired protein production, endoplasmic reticulum stress, and yeast cell death extensively reported previously [36,37]. Hence, rLys P. pastoris seems to be more suitable for S. aureus eradication from the environment or skin surfaces at temperatures around $33^{\circ} \mathrm{C}$. For in vivo applications based on oral probiotic administration, this methodology could be easily transferred to other non-pathogenic yeast strains displaying elevated growth temperatures. Several strains of Saccharomyces and Candida are culturable at temperatures up to $42{ }^{\circ} \mathrm{C}$, providing this option, while strict safety precautions must be taken into account.

An extensive number of cryptic AR enzymes yet to be discovered [38] and resistance toward lysostaphin was documented for laboratory strains [19,39]. The major mechanism of acquired lysostaphin resistance relates to mutations in the fem $A$ gene, which encodes the factor responsible for the addition of the second and third glycines to the pentaglycine bridge of the cell wall [40]. However, lysostaphin-resistant variants of $S$. aureus demonstrate reduced fitness in vitro and in vivo [41]. Moreover, resistance to lysostaphin is associated with a loss of resistance toward $\beta$-lactams, resulting from a change in the PG crossbridge from pentaglycine to a single glycine [42]. Hence, the application of rLys yeast biocontrol agents may provide an artificial selective pressure for the amplification of 
S. aureus strains sensitive to conventional $\beta$-lactam antibiotics, thus passively targeting $\beta$-lactam AR. The combination of rLys yeast with $\beta$-lactam antibiotics may be another straightforward option, since lysostaphin and $\beta$-lactams have synergistic effects on oxacillin-resistant Staphylococci [43].

The efficient eradication of $S$. aureus by live rLys P. pastoris was achieved at high scales, resulting in a simple and cost-effective strategy for $S$. aureus lysis in bioproduction and surface decontamination. The reported strategy is easily scalable, providing an almost unlimited source of the biocompatible S. aureus-killing agent. Live rLys P. pastoris cultures diluted more than 1000 folds efficiently eradicate S. aureus. The exceptional viability of yeast, in turn, prevents subsequent re-colonization, serving as a selective artificial biocontrol agent. Future biomedical applications based on designer yeast biocontrol agents require their evaluation in vivo. However, we believe that this strategy is very promising, since it provides highly safe, efficient and selective genetically programmed probiotics and targeted biocontrol agents.

\section{Materials and Methods}

\subsection{Bacterial and Yeast Strains}

A bacterial collection of clinical isolates including Enterococcus faecium, Enterococcus faecalis, Macrococcus caseolyticus, Staphylococcus vitulinus, Staphylococcus pasteuri, Staphylococcus warneri, Staphylococcus xylosus, Staphylococcus haemolyticus and Staphylococcus epidermidis was kindly provided by Lytech Co. Ltd. (Moscow, Russia). Escherichia coli BL21(DE3), Bacillus subtilis 168 ATCC 23857 and clinical isolates were used as indicator bacteria in activity tests. Staphylococcus aureus, constitutively producing GFP, was described previously [27]. Pichia pastoris GS115 was used as a heterologous host for secreted lysostaphin production. rLys P. pastoris and the control mCherry-producing strain were obtained from Pichia pastoris GS115, transformed with pGAP-rLys or pGAP-mCherry, respectively.

\subsection{Cultivation Conditions}

Pichia pastoris was cultured in a $2 \mathrm{YT}$ medium $(10 \mathrm{~g} / \mathrm{L}$ yeast extract, $16 \mathrm{~g} / \mathrm{L}$ tryptone, $5 \mathrm{~g} / \mathrm{L} \mathrm{NaCl})$ at $30{ }^{\circ} \mathrm{C}$ in an orbital shaker at $200 \mathrm{rpm}$. All bacteria were grown in $2 \mathrm{YT}$. The bacteria were inoculated with overnight culture using 1:100 dilutions, and were grown for $4 \mathrm{~h}$ at $37^{\circ} \mathrm{C}$ and $250 \mathrm{rpm}$ shaking. Chloramphenicol was added to a final concentration of $10 \mu \mathrm{g} / \mathrm{mL}$ for the $S$. aureus overnight culture.

\subsection{Plasmid Construction and Yeast Transformation}

Plasmid pGAPZalpha_BsmBI was constructed based on the pGAPZalpha A vector for the constitutive extracellular production of target proteins. An additional BsmBI restriction site was cloned into the MCS using annealed oligonucleotides. It enabled the insertion of a gene immediately downstream of the leader peptide. A codon-optimized lysostaphin sequence (GenBank: KF724949.1) was chemically synthesized and sub-cloned into the plasmid pGAPZalpha_BsmBI flanked by BsmBI/SalI restriction sites. The resulting plasmid pGAP-rLys was linearized by the AvrII restriction site and the electroporated P. pastoris GS115 strain using lithium acetate and dithiothreitol [44]. The same procedure was done for a similar pGAP-mCherry construct. Transformed cells were selected on YPDS plates $(2 \%$ peptone, $1 \%$ yeast extract, $2 \%$ glucose, $1 \mathrm{M}$ sorbitol, $2 \%$ agar) supplemented with $100 \mu \mathrm{g} / \mathrm{mL}$ of zeocin.

\subsection{Recombinant Lysostaphin Production}

The overnight culture of rLys P. pastoris was used to inoculate $100 \mathrm{~mL} 2 \mathrm{YT}$ to a final optical density at $600 \mathrm{~nm}\left(\mathrm{OD}_{600}\right)$ of 3 , corresponding to $1.5 \times 10^{8} \mathrm{CFU} / \mathrm{mL}$. The yeast culture was grown for $96 \mathrm{~h}$ in $750 \mathrm{~mL}$ shake flasks. Aliquots were collected every $24 \mathrm{~h}$, centrifuged at $5000 \mathrm{rpm}$ for $10 \mathrm{~min}$, and supernatants were stored at $-80^{\circ} \mathrm{C}$. Protein concentration was estimated using comparative protein densitometry with a VersaDoc Molecular Imager MP 4000 system, followed by Quantity One software quantification and a Bradford Protein Assay of samples obtained by repetitive concentration with Amicon Ultra $10 \mathrm{kDa}$ Centrifugal Filters. 


\subsection{Agar Overlay Assay}

The agar overlay assay was conducted using soft-agar $(5 \mathrm{~g} / \mathrm{L}$ yeast extract, $8 \mathrm{~g} / \mathrm{L}$ tryptone, $2.5 \mathrm{~g} / \mathrm{L}$ $\mathrm{NaCl}, 0.7 \%$ agar, $10 \mu \mathrm{g} / \mathrm{mL}$ chloramphenicol). Tested $P$. pastoris strains were grown for 2 days on $2 Y \mathrm{~T}$ agar plates at $30{ }^{\circ} \mathrm{C}$. Soft agar was melted, cooled to $42{ }^{\circ} \mathrm{C}$, and inoculated with $S$. aureus using 1:2000 overnight culture dilution. A quantity of $5 \mathrm{~mL}$ of liquid soft agar was used to overlay P. pastoris streaks. Plates were incubated overnight at $30^{\circ} \mathrm{C}$ after agar solidification.

\subsection{Estimation of Lysostaphin Activity in Culture Media}

The activity of culture media was tested in 96-well plates using serial dilutions in 2YT medium at $33{ }^{\circ} \mathrm{C}$ in a microplate shaker with $600 \mathrm{rpm}$ shaking. The tested bacteria were diluted in $2 \mathrm{YT}$ medium to a final $\sim 10^{6} \mathrm{CFU} / \mathrm{mL}$. MICs were determined as the lowest concentration of lysostaphin that inhibited the growth of tested bacteria after $16 \mathrm{~h}$ of incubation. MBCs were determined as the lowest concentration of lysostaphin that inhibited the growth of the tested bacteria after 7 days of incubation. The bacterial growth time course was monitored by $\mathrm{OD}_{600}$ using a Varioskan Flash multimode reader. The growth of $S$. aureus was monitored using GFP fluorescence and $\mathrm{OD}_{600}$.

\subsection{Cocultivation}

The cocultivation analysis included the following: (i) rLys P. pastoris activity spectrum, (ii) temperature dependence of rLys P. pastoris activity, (iii) S. aureus killing kinetics, and (iv) S. aureus eradication landscapes. Both (i) and (ii) indicator bacterial strains were diluted in $2 \mathrm{YT}_{\mathrm{T}}$ to $10^{6} \mathrm{CFU} / \mathrm{mL}$ in 96-well plates. P. pastoris cells were washed with fresh 2YT and added to bacteria using various yeast concentrations in the $10^{4}-10^{8} \mathrm{CFU} / \mathrm{mL}$ range. Plates were incubated in a microplate shaker at $33^{\circ} \mathrm{C}$ and $600 \mathrm{rpm}$ for 7 days. The bacterial growth was monitored using GFP fluorescence, $\mathrm{OD}_{600}$ and light microscopy. (ii) Plates were incubated at $20^{\circ} \mathrm{C}, 25^{\circ} \mathrm{C}, 30^{\circ} \mathrm{C}, 33^{\circ} \mathrm{C}$ and $37^{\circ} \mathrm{C}$ for 7 days. (iii) S. aureus-killing kinetics were determined in $750 \mathrm{~mL}$ flasks at $33^{\circ} \mathrm{C}$ with $200 \mathrm{rpm}$ shaking. A quantity of $50 \mathrm{~mL}$ of 2 YT was inoculated with microorganisms at different target:effector (S. aureus/rLys P. pastoris) titers: $10^{10} / 10^{7} \mathrm{CFU} / \mathrm{mL}, 10^{10} / 10^{6} \mathrm{CFU} / \mathrm{mL}, 10^{8} / 10^{6} \mathrm{CFU} / \mathrm{mL}$ and $10^{6} / 10^{6} \mathrm{CFU} / \mathrm{mL}$. mCherry-producing P. pastoris was used in a $10^{7} \mathrm{CFU} / \mathrm{mL}$ concentration as a negative control. Aliquots were collected after 0 , 12 and $24 \mathrm{~h}$ of incubation. Ten-fold serial dilutions were plated on 2YT agar plates supplemented with $10 \mu \mathrm{g} / \mathrm{mL}$ of chloramphenicol and incubated overnight at $37^{\circ} \mathrm{C}$ for $S$. aureus count. (iv) Eradication landscapes were obtained by cocultivation of $S$. aureus and rLys $P$. pastoris, using three-fold serial dilutions starting from $10^{10} \mathrm{CFU} / \mathrm{mL}$ and $10^{8} \mathrm{CFU} / \mathrm{mL}$, respectively. Plates were incubated at $25^{\circ} \mathrm{C}$, $33^{\circ} \mathrm{C}$ and $37^{\circ} \mathrm{C}$ for 5 days in a microplate shaker with $600 \mathrm{rpm}$ shaking. The bacterial growth was monitored using GFP fluorescence, $\mathrm{OD}_{600}$ and light microscopy. The viability of $S$. aureus was assayed by plating, as was described above in (iii).

\section{Conclusions}

The constitutive heterologous production of highly specific bacteriolytic protease lysostaphin in yeast Pichia pastoris provides an efficient biocontrol agent, specifically killing S. aureus in coculture. Live rLys $P$. pastoris cultures diluted more than 1000 folds efficiently eradicate $S$. aureus. A yeast-based anti-S. aureus probiotic was efficient in a high range of temperatures and target-to-effector ratios, indicating its robustness and versatility in eliminating $S$. aureus cells. The efficient eradication of $S$. aureus by live rLys $P$. pastoris was achieved at high scales, resulting in a simple and cost-effective strategy for $S$. aureus lysis in bioproduction and surface decontamination. These results illustrate the considerable potential of live lysostaphin-producing cultures in S. aureus elimination, creating a background for their further applications in vivo.

Author Contributions: I.V.S., A.G.G. and S.S.T. designed research; S.O.P., Y.A.M. and S.S.T. performed research; S.O.P. and S.S.T. analyzed data; I.V.S., A.G.G. and S.S.T. wrote the paper. All authors have read and agreed to the published version of the manuscript. 
Funding: This work was supported by Grant 18-29-08054 from the Russian Foundation for Basic Research and Grant 19-14-00331 from the Russian Science Foundation.

Conflicts of Interest: The authors declare no conflict of interest.

\section{References}

1. Fleischmann, C.; Scherag, A.; Adhikari, N.K.J.; Hartog, C.S.; Tsaganos, T.; Schlattmann, P.; Angus, D.C.; Reinhart, K. Assessment of Global Incidence and Mortality of Hospital-treated Sepsis. Current Estimates and Limitations. Am. J. Respir. Crit. Care Med. 2016, 193, 259-272. [CrossRef] [PubMed]

2. Rudd, K.E.; Johnson, S.C.; Agesa, K.M.; Shackelford, K.A.; Tsoi, D.; Kievlan, D.R.; Colombara, D.V.; Ikuta, K.S.; Kissoon, N.; Finfer, S.; et al. Global, regional, and national sepsis incidence and mortality, 1990-2017: Analysis for the Global Burden of Disease Study. Lancet 2020, 395, 200-211. [CrossRef]

3. Kourtis, A.P.; Hatfield, K.; Baggs, J.; Mu, Y.; See, I.; Epson, E.; Nadle, J.; Kainer, M.A.; Dumyati, G.; Petit, S.; et al. Vital Signs: Epidemiology and Recent Trends in Methicillin-Resistant and in Methicillin-Susceptible Staphylococcus aureus Bloodstream Infections-United States. MMWR Morb. Mortal. Wkly. Rep. 2019, 68, 214-219. [CrossRef] [PubMed]

4. Chambers, H.F.; DeLeo, F.R. Waves of resistance: Staphylococcus aureus in the antibiotic era. Nat. Rev. Microbiol. 2009, 7, 629-641. [CrossRef] [PubMed]

5. Turner, N.A.; Sharma-Kuinkel, B.K.; Maskarinec, S.A.; Eichenberger, E.M.; Shah, P.P.; Carugati, M.; Holland, T.L.; Fowler, V.G. Methicillin-resistant Staphylococcus aureus: An overview of basic and clinical research. Nat. Rev. Microbiol. 2019, 17, 203-218. [CrossRef] [PubMed]

6. Harris, S.R.; Feil, E.J.; Holden, M.T.G.; Quail, M.A.; Nickerson, E.K.; Chantratita, N.; Gardete, S.; Tavares, A.; Day, N.; Lindsay, J.A.; et al. Evolution of MRSA During Hospital Transmission and Intercontinental Spread. Science 2010, 327, 469-474. [CrossRef] [PubMed]

7. Ochman, H.; Elwyn, S.; Moran, N.A. Calibrating bacterial evolution. Proc. Natl. Acad. Sci. USA 1999, 96, 12638-12643. [CrossRef] [PubMed]

8. Gibson, B.; Wilson, D.J.; Feil, E.; Eyre-Walker, A. The distribution of bacterial doubling times in the wild. Proc. Biol. Sci. 2018, 285, 20180789. [CrossRef]

9. Waters, E.M.; Rowe, S.E.; O'Gara, J.P.; Conlon, B.P. Convergence of Staphylococcus aureus Persister and Biofilm Research: Can Biofilms Be Defined as Communities of Adherent Persister Cells? PLoS Pathog. 2016, 12, e1006012. [CrossRef]

10. Wu, J.A.; Kusuma, C.; Mond, J.J.; Kokai-Kun, J.F. Lysostaphin disrupts Staphylococcus aureus and Staphylococcus epidermidis biofilms on artificial surfaces. Antimicrob. Agents Chemother. 2003, 47, 3407-3414. [CrossRef]

11. Schindler, C.A.; Schuhardt, V.T. Lysostaphin: A New Bacteriolytic Agent for the Staphylococcus. Proc. Natl. Acad. Sci. USA 1964, 51, 414-421. [CrossRef]

12. Gonzalez-Delgado, L.S.; Walters-Morgan, H.; Salamaga, B.; Robertson, A.J.; Hounslow, A.M.; Jagielska, E.; Sabała, I.; Williamson, M.P.; Lovering, A.L.; Mesnage, S. Two-site recognition of Staphylococcus aureus peptidoglycan by lysostaphin SH3b. Nat. Chem. Biol. 2020, 16, 24-30. [CrossRef]

13. Bastos, M.D.; Coutinho, B.G.; Coelho, M.L. Lysostaphin: A Staphylococcal Bacteriolysin with Potential Clinical Applications. Pharmaceuticals 2010, 3, 1139-1161. [CrossRef] [PubMed]

14. Whitman, W.B. Staphylococcus. In Bergey's Manual of Systematics of Archaea and Bacteria; John Wiley \& Sons: Hoboken, NJ, USA, 2015; pp. 1-43. [CrossRef]

15. Kusuma, C.M.; Kokai-Kun, J.F. Comparison of four methods for determining lysostaphin susceptibility of various strains of Staphylococcus aureus. Antimicrob. Agents Chemother. 2005, 49, 3256-3263. [CrossRef] [PubMed]

16. Yang, X.Y.; Li, C.R.; Lou, R.H.; Wang, Y.M.; Zhang, W.X.; Chen, H.Z.; Huang, Q.S.; Han, Y.X.; Jiang, J.D.; You, X.F. In vitro activity of recombinant lysostaphin against Staphylococcus aureus isolates from hospitals in Beijing, China. J. Med. Microbiol. 2007, 56, 71-76. [CrossRef] [PubMed]

17. Placencia, F.X.; Kong, L.; Weisman, L.E. Treatment of methicillin-resistant Staphylococcus aureus in neonatal mice: Lysostaphin versus vancomycin. Pediatr. Res. 2009, 65, 420-424. [CrossRef] [PubMed]

18. Dajcs, J.J.; Hume, E.B.; Moreau, J.M.; Caballero, A.R.; Cannon, B.M.; O'Callaghan, R.J. Lysostaphin treatment of methicillin-resistant Staphylococcus aureus keratitis in the rabbit. Investig. Ophthalmol. Vis. Sci. 2000, 41, 1432-1437. 
19. Climo, M.W.; Patron, R.L.; Goldstein, B.P.; Archer, G.L. Lysostaphin treatment of experimental methicillin-resistant Staphylococcus aureus aortic valve endocarditis. Antimicrob. Agents Chemother. 1998, 42, 1355-1360. [CrossRef]

20. Kokai-Kun, J.F.; Chanturiya, T.; Mond, J.J. Lysostaphin as a treatment for systemic Staphylococcus aureus infection in a mouse model. J. Antimicrob. Chemother. 2007, 60, 1051-1059. [CrossRef]

21. Johnson, C.T.; Wroe, J.A.; Agarwal, R.; Martin, K.E.; Guldberg, R.E.; Donlan, R.M.; Westblade, L.F.; García, A.J. Hydrogel delivery of lysostaphin eliminates orthopedic implant infection by Staphylococcus aureus and supports fracture healing. Proc. Natl. Acad. Sci. USA 2018, 115, E4960-E4969. [CrossRef]

22. Kerr, D.E.; Plaut, K.; Bramley, A.J.; Williamson, C.M.; Lax, A.J.; Moore, K.; Wells, K.D.; Wall, R.J. Lysostaphin expression in mammary glands confers protection against staphylococcal infection in transgenic mice. Nat. Biotechnol. 2001, 19, 66-70. [CrossRef] [PubMed]

23. Liu, Y.; Bai, P.; Woischnig, A.K.; Charpin-El Hamri, G.; Ye, H.; Folcher, M.; Xie, M.; Khanna, N.; Fussenegger, M. Immunomimetic Designer Cells Protect Mice from MRSA Infection. Cell 2018, 174, 259-270. [CrossRef] [PubMed]

24. Blazanovic, K.; Zhao, H.; Choi, Y.; Li, W.; Salvat, R.S.; Osipovitch, D.C.; Fields, J.; Moise, L.; Berwin, B.L.; Fiering, S.N.; et al. Structure-based redesign of lysostaphin yields potent antistaphylococcal enzymes that evade immune cell surveillance. Mol. Ther. Methods Clin. Dev. 2015, 2, 15021. [CrossRef] [PubMed]

25. Zhao, H.; Blazanovic, K.; Choi, Y.; Bailey-Kellogg, C.; Griswold, K.E. Gene and protein sequence optimization for high-level production of fully active and aglycosylated lysostaphin in Pichia pastoris. Appl. Environ. Microbiol. 2014, 80, 2746-2753. [CrossRef] [PubMed]

26. Vollmer, W.; Blanot, D.; De Pedro, M.A. Peptidoglycan structure and architecture. FEMS Microbiol. Rev. 2008, 32, 149-167. [CrossRef]

27. Terekhov, S.S.; Smirnov, I.V.; Stepanova, A.V.; Bobik, T.V.; Mokrushina, Y.A.; Ponomarenko, N.A.; Belogurov, A.A.; Rubtsova, M.P.; Kartseva, O.V.; Gomzikova, M.O.; et al. Microfluidic droplet platform for ultrahigh-throughput single-cell screening of biodiversity. Proc. Natl. Acad. Sci. USA 2017, 114, 2550-2555. [CrossRef]

28. Terekhov, S.S.; Smirnov, I.V.; Malakhova, M.V.; Samoilov, A.E.; Manolov, A.I.; Nazarov, A.S.; Danilov, D.V.; Dubiley, S.A.; Osterman, I.A.; Rubtsova, M.P.; et al. Ultrahigh-throughput functional profiling of microbiota communities. Proc. Natl. Acad. Sci. USA 2018, 115, 9551-9556. [CrossRef]

29. Terekhov, S.S.; Nazarov, A.S.; Mokrushina, Y.A.; Baranova, M.N.; Potapova, N.A.; Malakhova, M.V.; Ilina, E.N.; Smirnov, I.V.; Gabibov, A.G. Deep Functional Profiling Facilitates the Evaluation of the Antibacterial Potential of the Antibiotic Amicoumacin. Antibiotics 2020, 9, 157. [CrossRef]

30. Watson, S.P.; Clements, M.O.; Foster, S.J. Characterization of the starvation-survival response of Staphylococcus aureus. J. Bacteriol. 1998, 180, 1750-1758. [CrossRef]

31. Parenteau, J.; Maignon, L.; Berthoumieux, M.; Catala, M.; Gagnon, V.; Abou Elela, S. Introns are mediators of cell response to starvation. Nature 2019, 565, 612-617. [CrossRef]

32. Petti, A.A.; Crutchfield, C.A.; Rabinowitz, J.D.; Botstein, D. Survival of starving yeast is correlated with oxidative stress response and nonrespiratory mitochondrial function. Proc. Natl. Acad. Sci. USA 2011, 108, E1089-E1098. [CrossRef] [PubMed]

33. Sideri, T.; Rallis, C.; Bitton, D.A.; Lages, B.M.; Suo, F.; Rodríguez-López, M.; Du, L.-L.; Bähler, J. Parallel profiling of fission yeast deletion mutants for proliferation and for lifespan during long-term quiescence. G3 2014, 5, 145-155. [CrossRef] [PubMed]

34. Rebnegger, C.; Vos, T.; Graf, A.B.; Valli, M.; Pronk, J.T.; Daran-Lapujade, P.; Mattanovich, D. Pichia pastoris Exhibits High Viability and a Low Maintenance Energy Requirement at Near-Zero Specific Growth Rates. Appl. Environ. Microbiol. 2016, 82, 4570-4583. [CrossRef] [PubMed]

35. Anderson, K.L.; Roberts, C.; Disz, T.; Vonstein, V.; Hwang, K.; Overbeek, R.; Olson, P.D.; Projan, S.J.; Dunman, P.M. Characterization of the Staphylococcus aureus Heat Shock, Cold Shock, Stringent, and SOS Responses and Their Effects on Log-Phase mRNA Turnover. J. Bacteriol. 2006, 188, 6739-6756. [CrossRef] [PubMed]

36. Li, Z.; Xiong, F.; Lin, Q.; d'Anjou, M.; Daugulis, A.J.; Yang, D.S.C.; Hew, C.L. Low-Temperature Increases the Yield of Biologically Active Herring Antifreeze Protein in Pichia pastoris. Protein Expr. Purif. 2001, 21, 438-445. [CrossRef] 
37. Zhong, Y.; Yang, L.; Guo, Y.; Fang, F.; Wang, D.; Li, R.; Jiang, M.; Kang, W.; Ma, J.; Sun, J.; et al. High-temperature cultivation of recombinant Pichia pastoris increases endoplasmic reticulum stress and decreases production of human interleukin-10. Microbial. Cell Factories 2014, 13, 163. [CrossRef]

38. Terekhov, S.S.; Mokrushina, Y.A.; Nazarov, A.S.; Zlobin, A.; Zalevsky, A.; Bourenkov, G.; Golovin, A.; Belogurov, A.; Osterman, I.A.; Kulikova, A.A.; et al. A kinase bioscavenger provides antibiotic resistance by extremely tight substrate binding. Sci. Adv. 2020, 6, eaaz9861. [CrossRef]

39. Strandén, A.M.; Ehlert, K.; Labischinski, H.; Berger-Bächi, B. Cell wall monoglycine cross-bridges and methicillin hypersusceptibility in a fem $\mathrm{AB}$ null mutant of methicillin-resistant Staphylococcus aureus. J. Bacteriol. 1997, 179, 9-16. [CrossRef]

40. Ehlert, K.; Schröder, W.; Labischinski, H. Specificities of FemA and FemB for different glycine residues: FemB cannot substitute for FemA in staphylococcal peptidoglycan pentaglycine side chain formation. J. Bacteriol. 1997, 179, 7573-7576. [CrossRef]

41. Kusuma, C.; Jadanova, A.; Chanturiya, T.; Kokai-Kun, J.F. Lysostaphin-resistant variants of Staphylococcus aureus demonstrate reduced fitness in vitro and in vivo. Antimicrob. Agents Chemother. 2007, 51, 475-482. [CrossRef]

42. Climo, M.W.; Ehlert, K.; Archer, G.L. Mechanism and suppression of lysostaphin resistance in oxacillin-resistant Staphylococcus aureus. Antimicrob. Agents Chemother. 2001, 45, 1431-1437. [CrossRef] [PubMed]

43. Kiri, N.; Archer, G.; Climo, M.W. Combinations of lysostaphin with beta-lactams are synergistic against oxacillin-resistant Staphylococcus epidermidis. Antimicrob. Agents Chemother. 2002, 46, 2017-2020. [CrossRef] [PubMed]

44. Wu, S.; Letchworth, G.J. High efficiency transformation by electroporation of Pichia pastoris pretreated with lithium acetate and dithiothreitol. BioTechniques 2004, 36, 152-154. [CrossRef] [PubMed]

(C) 2020 by the authors. Licensee MDPI, Basel, Switzerland. This article is an open access article distributed under the terms and conditions of the Creative Commons Attribution (CC BY) license (http://creativecommons.org/licenses/by/4.0/). 